\title{
Reproduce stylized facts of artificial financial market and comparison with real data
}

\author{
Yosra Mefteh Rekik, Mohamed Naceur Souissi \\ Department of Financial and Accounting Methods, Economics and Management University, Sfax, Tunisia
}

Received: January 27, 2015

Accepted: March 3, $2015 \quad$ Online Published: March 16, 2015

DOI: $10.5430 /$ air.v4n2p13

URL: http://dx.doi.org/10.5430/air.v4n2p13

\begin{abstract}
Agent-based computational models represent a big challenge in many disciplines. A vital approach receiving much interest is agent-based models, which gives a new area providing some ways to tackle some of the restrictions of the analytical models in finance. The aim of our research is to contribute to the behavioral finance and agent-based artificial markets by studying their market-wise implications using computational simulations. We investigate and analyze the behavioral foundations of the stylized facts of empirical data such as that characterize real data in financial markets. Our results confirm the existence of most the stylized facts such as leptokurtosis, non-independently distributed, and volatility clustering. From this attention, the artificial financial market will for all time be evaluated in order to have explication about market dynamics in Tunisian financial market.
\end{abstract}

Key Words: Tunisian stock market, Stylized facts, Behavior foundations, Agent based approach, Artificial stock market

\section{Introduction}

The agent-based simulation that deals with economic environments are sometimes referred to agent-based computational finance which obviously includes agent-based artificial market. ${ }^{[1]}$ Agent-based modeling offers many more parameters that can be altered to generate different scenarios. It also facilitates the study of emergent properties of traders' interactions and particular classes of traders in isolation. A key challenge for agent-based models is to demonstrate that the resulting price dynamics are indeed consistent with known empirical facts that remain difficult for more standard approaches in many disciplines. ${ }^{[2-4]}$ The prices of stocks and commodities in financial markets fluctuate over time which then produces financial time series. These time series are in fact of great attention for making inferences and predictions. By means of modern technologies, one can now obtain a vast amount of financial data that record every transaction in financial markets which was not explain by traditional models. ${ }^{[5,6]}$

Cont ${ }^{[5]}$ points out that those stylized facts should all be considered as mostly qualitative properties of returns since they may not be precise enough to differentiate between diverse parametric models. Nonetheless, even as qualitative, these properties can be quite constraining, since it is difficult to come up with stochastic processes or models that can produce a lot of them at the same time. To faithfully illustrate empirical data, more sophisticated models are needed. Recently, Panait and $\mathrm{Lupu}^{[7]}$ suggested some measures that could facilitate its sustainable development during the 20072009. Harrison et al. ${ }^{[8]}$ presented the empirical proprieties for the indices employing a panel data analysis. The authors pointed out some particular features of index returns in these types of markets: significant cross correlations and a high degree of non-linearity. Slavescu and Panait ${ }^{[9]}$ investigated

\footnotetext{
*Correspondence: Yosra Mefteh Rekik; Email: meftehyosra@yahoo.fr; Address: Department of Financial and Accounting Methods, Economics and Management University, Sfax, Tunisia.
} 
also the volatility of the daily returns for many companies during 2007-2011 listed on Bucharest Market Exchange and investigated the causal links with added international financial markets.

As an issue of fact, it may be essential to look deeper into the mechanisms of artificial stock markets in order to realize the complexity of price dynamics. Artificial computational simulations have become in the last few years a growing field of interest under the impulsion of the Santa-Fe-Institute. To study this complex system, we used approach of traders with bounded rationality that learn and make their behaviors change in time. According the origin study of Arthur ${ }^{[1]}$ and LeBaron (1995), which showed that it is probable to make rational global behaviors emerge with simple, bounded individual behaviors, several models of markets have been developed. The traders in the SFI-ASM differ from one another in the way they compute their anticipations about future prices and dividends from the similar information set by means of sets of rules that are a modified edition of John Holland's classifier system. The Santa-Fe-Institute artificial market is able to replicate some of the stylized facts of real financial markets.

The developing computational models, that forges analysis for more approaches in economic and finance to use multiagent analysis as one of its analytical tools for giving better comprehension of financial system. As shown in Figure 1, time-series data analysis giving a complete description of financial economy data properties. Yet, in the other side various arguments of the micro properties of financial economy agents tried to seek a linkage between macro analyses along with the behavior of traders.

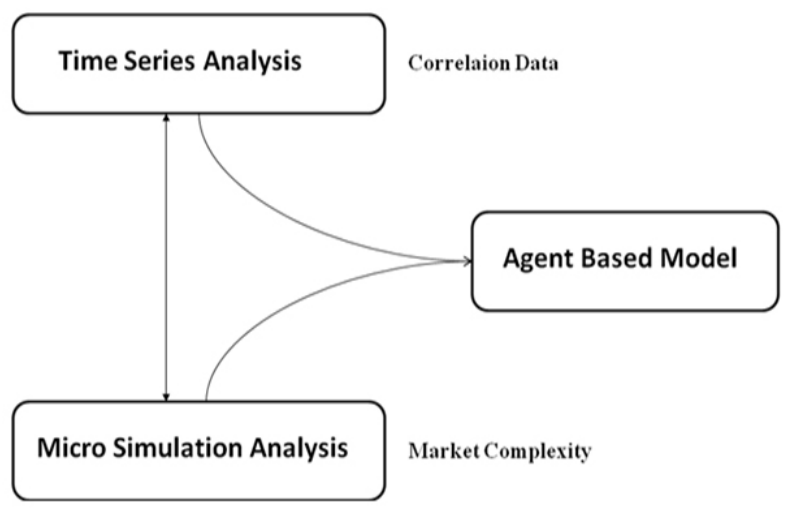

Figure 1: Connection between micro-simulation of financial system and its statistical features

How to clarify effects in macro-behavior in the situation of micro-interaction is the major motivation of using multiagent simulation models in social sciences, as well as financial economy. ${ }^{[10]}$

This paper is trying to give illustrations of multi-agents models that have been often utilized in computational ap- proach, continued with building primitive model from financial system with adaptive efforts upon stock market in Tunisia. The rest of this paper is planned as follow: In the second section, we review the important of such "stylized facts" emerge in the stock market. Section three deals with the description of the computational model that are going to be explored in order to obtain stylizes facts. The simulation results are shown in section four. In section five, we terminate with features of the computational model that lead to the reproduction some of the exhibited stylized facts of the assets return. Section six concludes and summarizes the research finding of this paper.

\section{Statistical properties of financial data}

In agent-based models of stock markets, it is standard practice to measure the validity of the model by investigating whether the stock price exhibits statistical features, known as the "stylized facts". Cont ${ }^{[5]}$ introduces various qualitative proprieties of logarithmic returns for financial assets. The proprieties investigated in studies of markets by Cont is sufficient to consider that his introduced stylized facts can be found in several types of financial data and for different types of financial markets. Important observed stylized statistical facts in financial time series are as follows:

- Absence of autocorrelations: (Linear) autocorrelations of returns are usually insignificant, except for very high-frequency data where microstructure starts playing a role.

- Volatility clustering: Non-linear functions of returns exhibit a positive autocorrelation. The time series justifying this are called "heteroscedastic". Thus, we can test whether a series is heteroscedastic with the Engle ARCH test.

- Aggregational Gaussianity: Specifically, the shape of the distribution is not the same at different time scales; and the time scale is increased (returns are calculated over a longer period), the more the distribution becomes normal.

- Leverage effect: The volatility of returns is negatively correlated with the returns themselves.

- Intermittency: At any time scale, returns display a high degree of variability.

- Volume/Volatility correlation: Trading volume is correlated with all measures of volatility.

- Gain/loss asymmetry: It is possible to observe one large downward in stock prices and stock index values but not equally large upward movements.

- Conditional heavy tails: Returns that have been corrected for volatility clustering still exhibit some degree of heavy tails. However, the tails are less heavy than in the unconditional distribution of returns. 
In order to prove that our endogenously generated price mimics some of the above described statistical properties similar with real data in Tunisian stock market, we will perform many sorts of test.

\section{Markets with agent-based models}

Agent-based computational finance is an emerging fields of research in which the researchers have build artificial markets that replicate characteristic behavior of regular markets, such as volume/volatility correlation, excess volatility, heavy tails of the distribution of daily and hourly returns and volatility clustering. To simplify our model, we choose the Walrasian auction, in which the equilibrium price is set so that overall demand equals overall supply, as found in the market clearing mechanism in price determination. ${ }^{[11-14]}$ Therefore, all trades will be executed at a fixed price and the agents are not permitted to trade out of equilibrium.

The artificial market programmed based on the Santa-Fe Artificial Stock Market and its modification was introduced in the papers of LeBaron, ${ }^{[15]}$ which contains two types of assets available for traders. One is the risk free asset paid with a fixed interest $r$ and the other is the risky asset paid at the beginning of each period, a stochastic dividend which is assumed to follows an auto-regressive process $\mathrm{AR}^{[1]}$ as:

$$
d_{t}=\bar{d}+\left[\rho\left(d_{t-1}\right)-\bar{d}\right]+\mu_{t}
$$

Where, $\mu t \sim N\left(0, \sigma^{2} \mu\right), \rho$ : exogenous parameter, $d_{t-1}$ dividend at $(t-1)$ and $\bar{d}$ is a mean of the autoregressive process. Agents maximize their utility function under the following constraint:

$$
W_{i, t+1}=x_{i, t}\left(p_{t+1}+d_{t+1}\right)-p_{t}\left(1+r_{f}\right)\left(W_{i, t}-p_{t} x_{i, t}\right)
$$

Where: $x_{i, t}$ is the number of risky assets detained by trader $i$ at time $t, r_{f}$ is the risk free interest rate, $d_{t+1}$ is the dividend attributed to risky assets, $p_{t}$ is the price at time period $(t)$.

It is well known that under CARA utility functions and Gaussian distribution for forecasts, traders' desire demand, $x_{i, t}$ for holding shares of the risky asset is linear in the expected excess return. ${ }^{[1]}$ According to Arthur et al., ${ }^{[1]}$ the demand of shares for agent $i$ at time $t$ is giving by:

$$
x_{i, t}=\frac{E_{i, t}\left(p_{t+1}+d_{t+1}\right)-p_{t}\left(1+r_{f}\right)}{\lambda \cdot \sigma_{i, t, p+d}^{2}}
$$

Where $\sigma_{i, t, p+d}^{2}$ is the conditional variance of $\left(p_{t+1}+d_{t+1}\right)$.

At equilibrium, the total demand must be equal to the total supply. Therefore, we assume that total supply equals the number of shares issued.

$$
\sum_{i=1}^{M} x_{i, t}=N
$$

The rational expectation equilibrium of future price and dividend are as follows:

$$
p_{t}=\beta \cdot \sum_{i=1}^{M} w_{i, t} E\left(p_{t+1}+d_{t+1}\right)-\alpha
$$

With:

$$
\beta=\frac{1}{\left(1+r_{f}\right)} ; w_{i, t}=\frac{\frac{1}{\sigma_{i, t, p+d}^{2}}}{\sum_{i=1}^{M} \frac{1}{\sigma_{i, t, p+d}^{2}}}=\beta \cdot \frac{\lambda N}{\sum_{i=1}^{M} \frac{1}{\sigma_{i, t, p+d}^{2}}}
$$

According to this expression, the equilibrium price depends on agents' expectations to the future value of the risky asset $E\left(p_{t+1}+d_{t+1}\right)$.

The stock market is composed by heterogeneous market participants (micro-interactions). In this view point, we can see that the price dynamics (macro-behavior) in the financial market proposed is emerged by the heterogeneous strategies of agents. ${ }^{[16-18]}$

\subsection{Fundamentalist traders}

We refer to traders who make investment decisions based on a supposed fundamental value as "fundamentalists". We describe a fundamental price by closely following the approach of Boswijk et al. ${ }^{[19]}$ The trade price is given as follows:

$$
p_{t}=\beta \cdot \sum_{i=1}^{M} w_{i, t} E\left(p_{t+1}+d_{t+1}\right)-\alpha
$$

With:

$$
\beta=\frac{1}{\left(1+r_{f}\right)} ; w_{i, t}=\frac{\frac{1}{\sigma_{i, t, p+d}^{2}}}{\sum_{i=1}^{M} \frac{1}{\sigma_{i, t, p+d}^{2}}}=\beta \cdot \frac{\lambda N}{\sum_{i=1}^{M} \frac{1}{\sigma_{i, t, p+d}^{2}}}
$$

\subsection{Noisy traders}

Investors have neither complete information nor infinite computational capacity. According to this behavioral bias, the expectation method used by noise traders is expressed as follows:

$$
\left\{\begin{array}{l}
p_{t+1}^{f}=p_{t}\left(1+\text { React }_{\text {Noisy }}\right) \\
d_{t+1}^{f}=d_{t}\left(1+\text { React }_{\text {Noisy }}\right)
\end{array}\right.
$$

Where: 


$$
\text { Reaction }_{N o i s y}=(1-k)+\left[1 / 5 \sum_{i=1}^{5}\left[\frac{p_{t+1}+d_{t-1}}{p_{t-1-i}}-1\right]\right]+K\left[1 / 5 \sum_{i=1}^{5}\left[\frac{p_{t-5}}{p_{t-5-i}}-1\right]\right]
$$

$K$ is the parameter measuring the weight of prior belief $(1 / 2$ $<K<1)$

$$
p_{t}=\beta \cdot \sum_{i=1}^{M} w_{i, t}\left[p_{t}\left(1+\operatorname{React}_{N o i s y}\right)+d_{t}\left(1+\operatorname{React}_{N o i s y}\right)\right]-\alpha
$$

with:

$\beta=\frac{1}{\left(1+r_{f}\right)} ; w_{i, t}=\frac{\frac{1}{\sigma_{i, t, p+d}^{2}}}{\sum_{i=1}^{M} \frac{1}{\sigma_{i, t, p+d}^{2}}} ; \alpha=\beta \cdot \frac{\lambda N}{\sum_{i=1}^{M} \frac{1}{\sigma_{i, t, p+d}^{2}}}$

\subsection{Loss-averse traders}

We create the model of the investors which is based on Prospect Theory in accordance with the one characteristic of decision making, stating that agents are more influenced by negative returns that positive returns. Like the rational, loss adverse investors use the artificial neural network to make forecasts but they may be reluctant to realize losses. Following Khaneman and Tversky's theory, loss adverse investors tend to estimate losses twice as large as profits. Thus, we stylize the utility function involving the loss aversion feature according to Shimokawa et al. (2007) as follows:

$$
U\left(w_{i, t+1}\right)=-e^{-\lambda B_{i, t} w_{L i, t+1}}
$$

$B_{i, t}$ is a loss Aversion coefficient:

$$
\begin{cases}B_{i, t}=4 & \text { if } E\left(p_{t+1}+d_{t+1}\right)-(p+d)_{t-1}^{r e f}<0 \\ B_{i, t}=1 & \text { if } E\left(p_{t+1}+d_{t+1}\right)-(p+d)_{t-1}^{r e f} \geq 0\end{cases}
$$

With $(P+d)_{t-1}^{r e f}$ is the reference point. It is measured by the average of price before $R$ periods. In others words, the gains and the losses are measured relative to reference point set to be the average of prices and dividend before 10 periods as follows:

$$
(p+d)_{t-1}^{r e f}=\frac{\left(p_{t}+d_{t}\right)+\left(p_{t-1}+d_{t-1}\right)+\cdots+\left(p_{t-R-1}+d_{t-R-1}\right)}{R}
$$

The expression of the equilibrium price victims' agents through loss aversion is formalized as follows:

$$
p_{t}=\beta \cdot \sum_{i=1}^{M} w_{i, t} E\left(p_{t+1}+d_{t+1}\right)-\theta
$$

With:

$$
\beta=\frac{1}{\left(1+r_{f}\right)} ; w_{i, t}=\frac{\frac{1}{\sigma_{i, t, p+d}^{2}}}{\sum_{i=1}^{M} \frac{1}{\sigma_{i, t, p+d}^{2}}} e t ; \theta=\beta \cdot \frac{\lambda N}{\sum_{i=1}^{M} \frac{1}{\sigma_{i, t, p+d}^{2}}}
$$

\section{Simulation results and analyses}

A computer simulation of the financial market involving 100 investors was used as the model in this paper; risk assets and risk free assets are the two possible transaction models which are adopted along with other behavioral features. ${ }^{[1,11-13]}$ As a review of the model, we can see Table 1 showing the parameters values used in simulations.
Table 1: Parameters list

\begin{tabular}{ll}
\hline Symbol & Explanation \\
\hline$M$ & The number of investors (100) \\
$N$ & The number of issued stocks (100) \\
$T$ & Simulation periods (1000) \\
$r_{f}$ & The risk free interest rate (0.1) \\
$\lambda$ & Risk averse ratio of the investor (0.5, constant) \\
$K$ & Measuring the weight of prior belief (0.8) \\
$\Lambda$ & Exogenous parameter (0.95) \\
$\overline{\mathrm{d}}$ & Mean of autoregressive process \\
$\sigma^{2}{ }_{\mu}$ & Conditional variance of dividend process $(0.0734)$ \\
$\theta$ & Forecast error update's parameter (0.013) \\
$f$ & $(6.333)$ \\
$g$ & $(16.688)$ \\
\hline
\end{tabular}

There are numerous design matter regarding the traders, as is described in Grothmann: ${ }^{[20]}$ the investment decision making process, heterogeneous strategies and learning. ${ }^{[21,22]}$ Thus, we assume the application of neural network so that fundamentalist learns the equilibrium parameters with rational expectations provided by the following equation: 


$$
E\left(p_{t+1}+d_{t+1}\right)=\rho\left(p_{t}+d_{t}\right)+(1-\rho)[(1+f) \bar{d}+g]
$$

Nero-one is used to develop the back-propagation learning algorithm. After several tests of varying the number of neurons in the hidden layer, we found that three neurons constitute the optimal structure of the hidden layer (see Table 2). Thus, we have simulated expectations for 1000 observations. As shown, the convergence of the artificial neural network to the rational expectations occurs at quadratic mean error $10^{-5}$.

Table 2: Artificial Neural Network performance criteria

\begin{tabular}{llll}
\hline Number of hidden layers & RMSE & $\mathbf{R}^{2}$ & $\mathbf{P E}$ \\
\hline 2 & $4.70 \mathrm{E}-05$ & 0.99 & $3.09 \mathrm{E}-05$ \\
$\mathbf{3}$ & $\mathbf{1 . 4 6 E - 0 5}$ & $\mathbf{1}$ & $\mathbf{9 . 9 2 E - 0 6}$ \\
4 & $2.45 \mathrm{E}-05$ & 1 & $1.66 \mathrm{E}-05$ \\
5 & $2.26 \mathrm{E}-04$ & 1 & $1.50 \mathrm{E}-04$ \\
\hline
\end{tabular}

The architecture of the neural network is composed of a single input layer, containing a single neuron, namely the vector $\left(P_{t}+d_{t}\right)$, an output layer, indicating the results forecasts $E\left(p_{t+1}+d_{t+1}\right)$ and three neurons of hidden layers. The aim of artificial invertors is to build the explication of the future price and dividend $E\left(p_{t+1}+d_{t+1}\right)$ which will be used in their demand functions. By means of feed-foreword Artificial Neural Network, we see in Figure 2 the expectations of future price and dividend versus rational expectations.

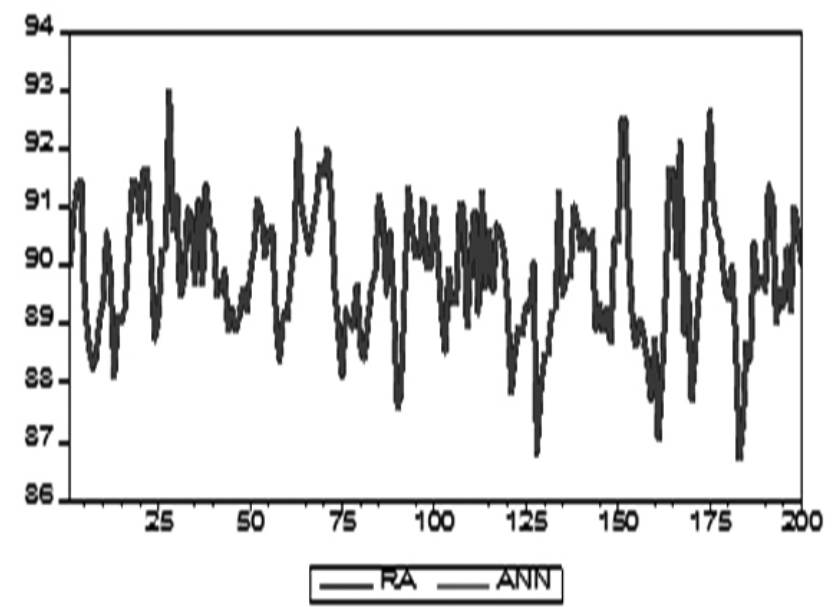

Figure 2: Rational vs. Artificial Neural Network Expectations

The graphical comparison shows the same graph on the desired results and those provided by the neural network. The graph in Figure 2 illustrates the dynamic simulation of the forecast price and future dividends. To run simulation, we use the same parameter values as used by LeBaron. ${ }^{[15]}$ The results show a correlation between the predictions made by the neural network and predictions under the assumption of rational expectations. In a second experiment, we consider Published by Sciedu Press the possible combination of behavioral investors in our artificial market simulations and examine whether the combinations explain more the assets prices dynamics. We combine fundamentalist, noise traders and loss aversion behavior affecting the prices dynamics.

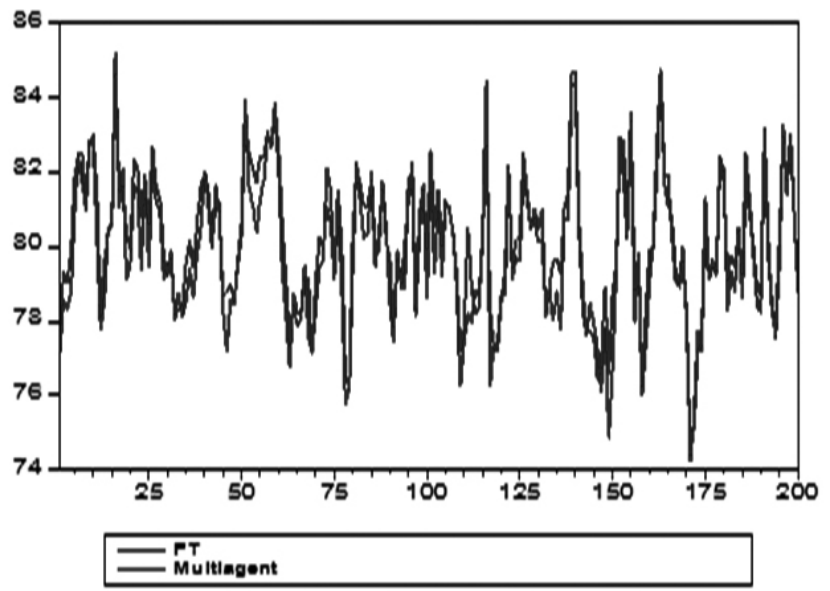

Figure 3: Experimental result with multi-agent simulation

According to the modeling and multi-agent simulation of our model, the combination of several types of agents with different proportions caused a small gap in their basic assets prices. From Figure 3, we can observe that the market price (blue line) fluctuates closely around the behavior of the market price value (red line). By varying the percentage of agents in the market, we have elucidated the impact of the degree of heterogeneity of investors on the price dynamics. This experiment adds another layer of complexity by allowing heterogeneity among of investors. This result confirms many studies which assume that heterogeneous agentbased models have included heterogeneity behavior originating among artificial agents. ${ }^{[16,23,24]}$

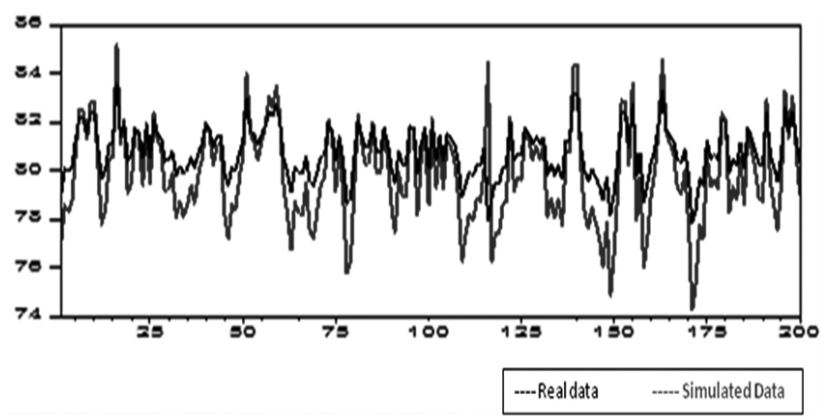

Figure 4: The simulation result vs. TUNINDEX returns

The simulation resulting (see Figure 4) shows that the agents in Tunisian stock market are truly bounded by the movement of the market. We report the artificial financial market that emerges the similar dynamics price with real data. Afterwards observing the price behavior, we will perform differ- 
ent sorts of statistical tests to identify if the price presents the stylized facts.

\section{Stylized facts in financial return}

In recent years, an important challenge of the financial approach is to construct more sophisticated models which have consistencies with as many financial proprieties that cannot be explained by traditional theory. We have constructed the artificial market to see how that emerges the analogous stylized facts with the real one for an individual but dominant index in Tunisian financial markets.

\section{Fact 1: Distribution of Return}

Our aim is to highlight the specificities of the Tunisian stock market through these various estimations. Descriptive statistics are estimated for Tunisian stock market (see Table 3). Return distribution shows a small negative skewness, a relative fatter right tail, and very large kurtosis (leptokurtic), clearly peaking above the normal distribution. Furthermore, the JB statistic confirms strong rejection of the null hypothesis of normality for financial returns. Table 3 shows that the value of the Jarque and Bera statistic is greater than the critical value for all the stocks of our sample. This result indicates that the distribution of the TUNINDEX returns is leptokurtic.

Table 3: TUNINDEX returns

\begin{tabular}{ll}
\hline Descriptive & Statistical \\
\hline Mean & $-1.94 \mathrm{e}-05$ \\
Median & 0.000000 \\
Maximum & 0.005246 \\
Minimum & -0.005282 \\
Std. Dev. & 0.001039 \\
Skewness & -0.060051 \\
Kurtosis & 6.632689 \\
Jarque-Bera & 6702.630 \\
Probability & 0.000000 \\
\hline
\end{tabular}

Table 3 shows that some descriptive statistics of the TUNIN-
DEX returns, from which one can see the fat-tailed character of the returns quite clearly. The normality hypothesis is definitively rejected because of asymmetry and kurtosis excess.

We observe in our simulation data in Figure 5 that the distribution of return also follows considering quite clearly the fat-tailed character of the returns. The values of the JarqueBera statistics confirm that the distribution of asset returns is significantly different from the normal distribution. The normal asymmetry coefficient or Skewness is generally different from zero and negative $(-1.389803)$ indicating that the asymmetry of the distribution series is different from that of the normal distribution. The coefficient of kurtosis is very high, well above 3 (17.15963). This confirms the "fats tails" observed in our artificial market.
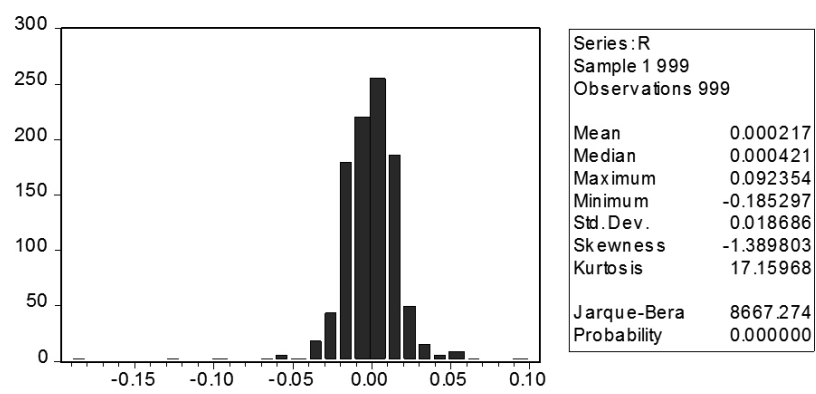

Figure 5: Histogram of simulation results

A quantile per quantile comparison of the empirical distribution with a theoretical normal can detect differences between the distributions of the simulated return compared with the real data (see Figure 6). This is another technique rather than the measurement of the skewness and kurtosis, in order to highlight more significantly the presence of fat tails. In our case, this is an analysis of quantiles, plotting empirical quantiles against the quantiles of the normal distribution. If distributions are identical, the plot should be approximately linear. However, if they are different, we should observe the deviations. The distribution of the TUNINDEX return is leptokurtic.

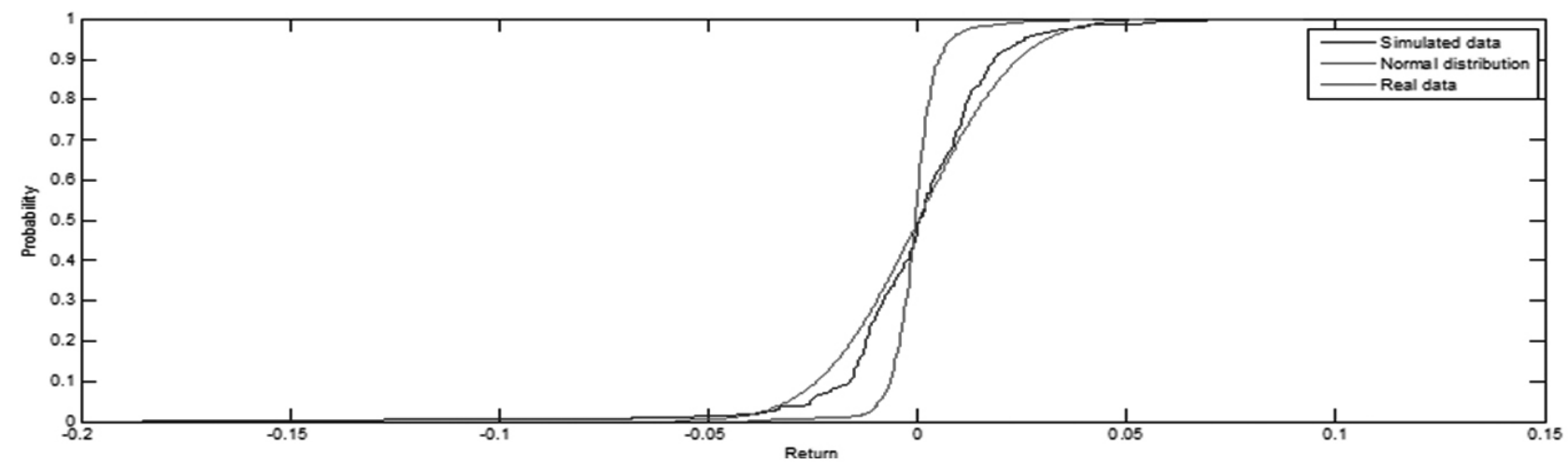

Figure 6: Q-Q Plot 


\section{Fact 2: Excessive Volatility}

Several studies in finance use autoregressive conditional heteroskedastic $(\mathrm{ARCH})$ models introduced in $1982,{ }^{[25]}$ the conditional variance of shocks, $h_{t}$, is a linear function of past squared values of the process:

$$
h_{t}=\omega+\alpha_{1} \varepsilon_{t-1}^{2}
$$

where $\omega>0$ and $\alpha_{1}>0$.

An alternative and more flexible structure is provided by Bollerslev $^{[26]}$ which is well known as a GARCH model. In its simplest representation, a GARCH $(1,1)$ model, is defined by:

$$
h_{t}=\omega+\alpha_{1} \varepsilon_{t-1}^{2}+\beta_{1} h_{t-1}
$$

We use ARCH to test for volatility in our agent-based model. The results are shown in Table 4 and Table 5. Table 4 shows that there are ARCH effects of the return of the fundamental value while from Table 5 we can see that there are GARCH effects of returns of the market price.

Table 4: ARCH effect

\begin{tabular}{|l|l|l|l|}
\hline \multicolumn{4}{|c|}{ ARCH test } \\
\hline F-statistic & 474.0514 & $p$-Value & 0.00 \\
\hline Obs R-squared & 772.8457 & $p$-Value & 0.00 \\
\hline
\end{tabular}

Table 5: GARCH test for market price

\begin{tabular}{|l|l|l|l|}
\hline \multicolumn{4}{|c|}{ GARCH test } \\
\hline F-statistic & 474.0514 & $p$-Value & 0.00 \\
\hline Obs R-squared & 321.9312 & $p$-Value & 0.00 \\
\hline
\end{tabular}

The ARCH-GARCH methodology reflects instability of volatility in time. The reference scheme is an autoregressive scheme in which we allow for the conditional standard deviation to evolve endogenously. We notice that it is about a specification that takes into account some stylized facts like persistence of financial returns.

\section{Fact 3: Volatility Clustering}

The pattern that we want to explore is the fact of volatility clustering in which large changes tend to follow large changes, and small changes tend to follow small changes. ${ }^{[25]}$ The volatility clustering is one of the important stylized facts of finance time series data. The cause of this property is the interaction between the heterogeneous investors; in our case: the fundamentalists, the noise traders and the loss adverse traders.

As showed in Figure 7 is remarkable so is indeed generated by the investors' trading just as observed in real markets. As a consequence, we find that the price formation and the statistical properties of the simulated return were closer to the dynamics and properties of the real financial data.

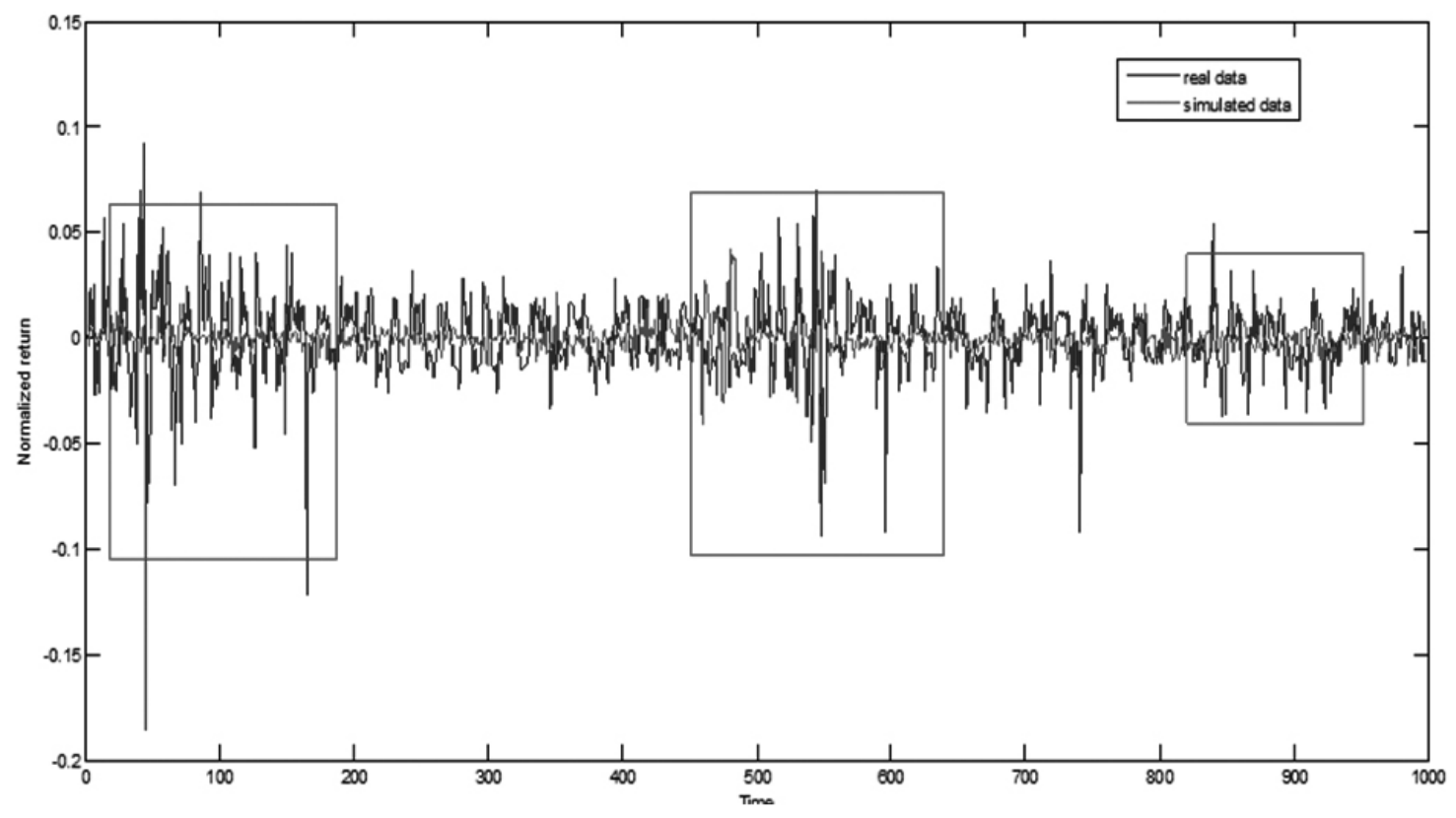

Figure 7: The Volatility Clustering

The conclusions derived from our research generally confirm the results of prior papers conducted by different researchers. Martinez-Jaramillo ${ }^{[22]}$ point out that fat tails, autocorrelation and volatility can be main factors to test the validity of the agent-based models. Liu et al., ${ }^{[27]}$ show that investors' switching between chartist and fundamentalist strategies is the main reason that lead to volatility clustering and the emergence of fat-tailed returns. Similar results 
are present in most financial data. ${ }^{[14,28]}$

As noted above, we show that the investors in Tunisian financial market are truly bounded by the movement of the market, since the tuning on the variable effect very sharply on the comparison to the real data. Our results illustrate that our artificial stock market is able to replicate several statistical proprieties observed in real one. In another hand, by knowledge on our structure of artificial market, we can find an important explication about market process in Tunisian Stock Exchange.

\section{Summary}

Recently, different structural models try to explain the complex behaviors of financial markets have received much attention. Artificial Intelligence as well as in exacting computational paradigm have been used to solve several financial problems. The heterogeneous agent literature allow for computational models which can reveal the causal dynamics in a world comprised of a number of heterogeneous agents which interact trough some trading mechanism, while possibly learning and evolving. Specifically, agents in real financial market base their current behavior partly on their past experience and partly on perceived market characteristics, which their past individual behavior has helped to determine. Particularly, the multi-agent models describing the artificial financial market are expected to offer us a way to analyze the behavior of agents in the market.

The preliminary results of this paper show that the artificial market approach is effective for understanding real financial markets. Based on the heterogeneous beliefs in artificial, we can make a support system for decision making in terms of market dynamics. With this agent-based artificial financial market model, we could make many further researches. For example, we could analyze the possible effects if a new type of investors joins in the market. Alternative, we can analyze the effects if a certain type of investors changes the decision making principle. These researches will be helpful for us to predict the possible changes to the dynamic financial markets.

\section{References}

[1] Arthur B. Inductive Reasoning and Bounded Rationality: The ElFarol Problem. American Economic Review. 1994; 84(2): 406-417.

[2] Farmer D, Joshi S. The Price Dynamics of Common Trading Strategies. Journal of Economic Behavior and Organization. 2002; 49(2): 149-171. http://dx.doi.org/10.1016/S0167-2681(02)000 65-3

[3] Cont R. Volatility Clustering in Financial Markets: Empirical Facts and Agent-Based Models in Long Memory in Economics. Springer. 2007: 289-310.

[4] Lux T, Westerhoff F. Economics Crisis. Nature Phys. 2009; 5: 2-3. http://dx.doi.org/10.1038/nphys 1163

[5] Cont R. Empirical properties of asset returns: stylized facts and statistical issues. Quantitative Finance. 2001; 1(2): 223-236. http: //dx.doi.org/10.1080/713665670

[6] Chakraborti A, Tokea M, Patriarca M, et al. Empirical facts and agent-based models. Econophysics. 2009.

[7] Panait I, Lupu I. The behavior of the Bucharest Stock Exchange during the Current Financial Markets Crisis and Proposed Measures for its Sustainable Development. Spiru Haret University Annales. Economic Series. 2009; 1(1): 73-80.

[8] Harrison B, Lupu R, Lupu I. Statistical Properties of the CEE Stock Market Dynamics: A Panel Data Analysis. The Romanian Economic Journal. 2010; (37): 41-54.

[9] Slăvescu EO, Panait I. Volatility and Causality Study of the Daily Returns on the Bucharest Stock Exchange during 2007-2011, published in Proceeding of the 17th International Conference The Knowledge-Based Organization-Economic. 2001: 292-300.

[10] Axtell R. Why Agents? On the varied Motivations for Agent Computing in The Social Sciences. Working Paper. 2000; 17.

[11] Grossman S, Stiglitz J. On the Impossibility of Informationally Efficiency Markets. American Economic Review. 1980; 70(3): 393-408.

[12] Hussman J. Market Efficiency and Inefficiency in Rational Expectations Equilibria: Dynamic Effects of Heterogeneous Information and Noise. Journal of Economic Dynamics and Control. 1992; 16: 655-680. http://dx.doi.org/10.1016/0165-1889(92) 9 0053-H
[13] Pajares J, Hernandez C, Lopez A. Modelling Learning and rd in Innovative Environments: A Cognitive Multi-Agents Approach. Journal of Soc. Soc. Simulation. 2005; 46(1): 1-30.

[14] Jiri K, Jozef B. Behavioral Breaks in the Heterogeneous Agent Model: The Impact of Herding, Overconfidence, and Market Sentiment. Statistical Mechanics and its Applications. 2013; 392 (23): 5920-5938. http://dx.doi.org/10.1016/j.physa. 201 3.07 .050

[15] LeBaron B. Building the Santa Fe Artificial Stock Market. Working Paper, Brandeis University. 2002.

[16] Lux T, Marchesi M. Volatility Clustering in Financial Markets: A Micro-simulation of Financial Agents. International journal of theoretical and applied finance. 2000; 3: 675-702. http://dx.doi.o rg/10.1142/S0219024900000826

[17] Ehrentreich N. Technical Trading in the Santa Fe Institute Artificial Stock Market Revisited. Journal of Economic Behavior and Organization. 2006; 61(4): 599-616. http://dx.doi.org/10.1016/j .jebo. 2004.07.022

[18] Franke R, Westerhoff F. Structural Stochastic volatility in asset pricing dynamics: estimation and model contest. Journal of Economic Dynamics and Control. 2012; 36: 1193-1211. http://dx.doi.o $\mathrm{rg} / 10.1016 / \mathrm{j} \cdot \mathrm{jedc} .2011 .10 .004$

[19] Boswijk P, Hommes C, Manzan S. Behavioral Heterogeneity in Stock Prices. Journal of Economic Dynamics and Control. 2007; 31: 1938-1970. http://dx.doi.org/10.1016/j.jedc. 2007.0 1.001

[20] Grothmann R. Multi-Agent Market Modeling Based on Neural Networks. PhD thesis.

[21] Chen H, Yeh H. On the Emergent Properties of Artificial Stock Markets: co-evolutionary gp-based Financial Market. IEEE Transactions on Evolutionary Computation. 2007.

[22] Martinez-Jaramillo S. Artificial Financial Markets: An Agent Based Approach to Reproduce Stylized Facts and to study the Red Queen Effect. Ph.D. thesis, Centre for Computational Finance and Economic Agents, University of Essex. 2007.

[23] Alfarano S, Wagner F, Milaković M. What Distinguishes Individual Stocks from the Index? The European Physical Journal-Condensed 
Matter and Complex Systems. 2010; 73: 23-28. http://dx.doi.o $\mathrm{rg} / 10.1140 / \mathrm{epjb} / \mathrm{e} 2009-00358-1$

[24] Manahov V, Robert H. Herd Behavior Experimental Testing in Laboratory Artificial Stock Market Settings: Behavioral Foundations of Stylised Facts of Financial Returns. Physica A: Statistical Mechanics and its Applications. 2013; 392(19): 4351-4372. http: //dx.doi.org/10.1016/j.physa.2013.05.029

[25] Engel F. Autoregressive Conditional Heteroscedasticity with Estimates of the Variance of United Kingdom Inflation. Econometrica. 1928; 50(4): 987-1007.
[26] Bollerslev T. Generalized Autoregressive Conditional Heteroskedasticity. Journal of Econometrics. 1986; 31(3): 307-327. http://dx.doi.org/10.1016/0304-4076(86) 90063-1

[27] Liu Y, Chao X, Zhang W, et al. Impact of information cost and switching of trading strategies in an artificial stock market. CNRS, Centre d'Economie de la Sorbonne. 2013: 1-12.

[28] Brock W, Hommes C. Heterogeneous Beliefs and Routes to Chaos in a Simple Asset Pricing Model. J. Econ. Dyn. Control. 1998; 22: 1235-1274. http://dx.doi.org/10.1016/S0165-1 889(98) 00011-6 